\title{
A FACTORIZATION THEOREM FOR GROUPS AND LIE ALGEBRAS
}

\author{
EUGENE SCHENKMAN*
}

\begin{abstract}
A proof is given for a generalization of a theorem of Mennicke that each member of a certain family of groups defined by generators and relations is finite. This leads to the following theorem on factorization of groups.

TheOREM. Let $G$ be generated by abelian subgroups $A, B, C$, such that $[A, B]<A,[B, C]<B,[C, A]<C$; then the second derived group, $G^{\prime \prime}$ is nilpotent of class at most 3 .
\end{abstract}

Also proved is the analogue of the above theorem for Lie algebras.

Mennicke [1] gave the first example of a finite 3-generator 3-relator group. In particular he studied the group $G_{k k k}$ as defined in Theorem 1 below. In studying Mennicke's examples we observe some interesting general results about factorizations by abelian groups. Since these depend only on the commutator calculus and since the Witt identity (cf. [2, p. 201]) is similar to the Jacobi identity we are led also to a result on Lie algebras (Theorem 2 below).

Our first theorem is mentioned without proof in the last paragraph of [1]. We use the notation $\langle M \mid N\rangle$ (also $\left\langle M \mid N=N_{1}\right\rangle$ ) for the group given by the set of generators denoted by $M$ and with the relators $N$ (or relations $N=N_{1}$ ). For $K \subseteq G,\langle K\rangle$ will denote the subgroup of $G$ generated by $K$.

TheOREM 1. For $r, s, t>1$ let $G_{r s t}$ denote $\langle a, b, c,| a^{b}=a^{r}, b^{c}=b^{s}$, $\left.c^{a}=c^{t}\right\rangle$. Then $G_{r s t}$ is finite.

Proof. If one of $a, b, c$ is finite then so are the other two in turn; this follows from the defining relations. Then since $\langle a, b\rangle$ is the split extension $[A] B=B A$ with $A$ denoting $\langle a\rangle, B$ denoting $\langle b\rangle$, and since $\langle b, c\rangle=[B] C=$ $C B(C$ denotes $\langle c\rangle)$, and since $\langle c, a\rangle=[C] A=A C$, it follows that $G=$ $A B C$ and is finite. Thus the theorem is proved if we show that one of $a, b, c$, is finite. Accordingly, in what follows we assume that each of $a, b, c$ has infinite order.

Now the group $G_{r}=\left\langle a, b \mid a^{b}=a^{r}\right\rangle$ is a split extension $[A]\langle b\rangle$ with $\langle b\rangle$ infinite cyclic and $A$ a locally cyclic group, since for $n$ a positive integer

Received by the editors April 1, 1977.

AMS (MOS) subject classifications (1970). Primary 20F05, 17B65; Secondary 20E15.

Key words and phrases. Generators, relations, factorization, abelian subgroup, abelian subalgebras, derived subgroup, derived subalgebra, nilpotent.

*This paper is being published posthumously. Professor Schenkman died on October 10, $197 \%$

C American Mathematical Society 1978 
$\left\langle a^{b^{n}}\right\rangle\langle\langle a\rangle$ and for $m, n$ any integers with $m\rangle n\left\langle a^{b^{m}}\right\rangle\left\langle\left\langle a^{b^{n}}\right\rangle\right.$ (we see this latter by conjugating with a suitable $b^{t}$ ). It follows easily that $A$ is isomorphic to the subgroup of the additive rationals $\left\{m / r^{n}\right\}$ with denominators a power of $r$. Any normal subgroup of $G_{r}$ contains an element $b_{0} a_{0}$, with $b_{0} \in\langle b\rangle, a_{0} \in A$, and its conjugate by $b,\left(b_{0} a_{0}\right)^{b}$; hence it contains $a_{0}^{-1} a_{0}^{b}$ which is an element of the form $a^{m r^{n}}$ (if $a_{0}=1$, consider $b_{0}^{ \pm 1}=b^{t}, t>0$, and $\left.b^{-t} \cdot a b^{t} a^{-1}=a^{r^{t}-1}\right)$. It follows that in every proper homomorphic image of $G_{r}$ the image of $a$ is finite. Since $a$ has infinite order by our assumptions, we conclude that the subgroup $\langle a, b\rangle$ of $G_{r s t}$ is isomorphic to $G_{r}$.

Now in $G_{r s}, a^{\left(b^{c}\right)}=a^{\left(b^{\prime}\right)}=a^{q}$ with $q=r^{s}$. Transforming this by $c^{-1}$ we get that $\left(a^{c^{-1}}\right)^{b}=\left(a^{q}\right)^{c^{-1}}=\left(a^{c^{-1}}\right)^{q}$. Thus $b$ normalizes $\left\langle a^{c^{-1}}\right\rangle$ as well as $\langle a\rangle$. We should note parenthetically that $b$ normalizes $\langle a\rangle$ since $\langle a\rangle^{b} \leqslant\langle a\rangle$, but $b$ is not in the normalizer of $\langle a\rangle$ since $\langle a\rangle^{b} \neq\langle a\rangle$. This is related to the fact that $a^{b^{-1}}=a^{1 / r} \notin\langle a\rangle$. We return to the subgroup $\langle a, c\rangle$ of $G_{r s t}$ which has the form $[C]\langle a\rangle$ isomorphic to $G_{t}$ (just as $\langle a, b\rangle \cong G_{r}$ ) and note that since $b$ normalizes $\langle a\rangle$ and $\left\langle a^{c^{-1}}\right\rangle, b$ normalizes a subgroup $\left[C_{0}\right]\langle a\rangle$ of $\langle a, c\rangle$ with $1<C_{0}<C$. Thus there is an element $c^{m t^{n}} \neq 1$ (with $n \geqslant 0$ ) in $C_{0}$ and hence its $t^{n}$ th power $c^{m} \in C_{0}$. Then $\left[C^{m}, b\right] \in C$. On the other hand, we have $b^{c}=b^{s}$ and hence $\left(c^{m}, b\right] \in B$ (with $\left.[B]\langle c\rangle \cong G_{s}\right)$. It follows that there is a power of $b$ equal to a power of $c$ and hence the automorphism induced by $b$ on $C$ is finite. Thus $c$ is finite contrary to our assumption that $c$ has infinite order. This proves the theorem.

An examination of the above theorem leads us to study a group $G$ generated by abelian subgroups $A, B, C$ with $\langle A, B\rangle=[A]=B A$ or equivalently $[A, B] \leqslant A$ and with $[B, C] \leqslant B,[C, A] \leqslant C$. Finite groups generated by pairwise commuting abelian subgroups were studied by Wielandt, and finite groups generated by pairwise commuting nilpotent subgroups were studied by Wielandt and also by Kegel, culminating in the theorem that such a group is solvable (cf. [2, p. 273, Theorem IX.2.f]). For infinite groups results are harder to obtain since the Sylow theory is not available. Prior to proving the theorem for groups, we prove the analogous theorem for Lie rings which may be illuminating.

We recall that in a Lie ring $L$ the product of two elements $a$ and $b$ is denoted by the bracket $[a, b]$; this product satisfies $[a, b]=-[b, a]$ and also satisfies the Jacobi identity $[[a, b], c]+[[b, c], a]+[[c, a], b]=0$. An abelian Lie ring $L$ satisfies $[L, L]=0$.

THEOREM 2. Let $A, B, C$ be abelian subrings of a Lie ring $L$ such that $L=A+B+C$ and $[A, B] \leqslant A,[B, C] \leqslant B,[C, A] \leqslant C$. Then $L^{\prime \prime}=$ $\left[L^{2}, L^{2}\right]$ is nilpotent of class at most 3 .

Proof. Let $L_{1}=A_{1}+B_{1}+C_{1}$ where $A_{1}, B_{1}, C_{1}$ are respectively the maximal ideals of $L$ contained in $A, B, C$. Then

$$
\begin{aligned}
L_{1}^{2} & =\left[L_{1}, L_{1}\right] \leqslant\left[A_{1}, B_{1}\right]+\left[B_{1}, C_{1}\right]+\left[C_{1}, A_{1}\right] \\
& \leqslant\left(A_{1} \cap B_{1}\right)+\left(B_{1} \cap C_{1}\right)+\left(C_{1} \cap A_{1}\right) .
\end{aligned}
$$


Furthermore $L_{1}^{3}=\left[L_{1}^{2}, L_{1}\right] \leqslant A_{1} \cap B_{1} \cap C_{1} \leqslant A \cap B \cap C$ which is central in $L$. Thus $L_{1}$ is nilpotent of class at most 3 .

Now $L^{\prime}=[A+B+C, A+B+C] \leqslant[A, B]+[B, C]+[C, A]$, and $L^{\prime \prime}$ $=\left[L^{\prime}, L^{\prime}\right]<[[A, B],[B, C]]+[[A, B],[C, A]]+[[B, C],[C, A]]$. For $a \in A$, $b \in B, c \in C$ we get from the Jacobi identity that $[[a, b], c]+[b, c], a+$ $[[c, a], b]=0$ with the first summand in $C$, the second in $A$, the third in $B$. Bracketing the above equation with $b_{1} \in B$ we get

$$
\left[[[a, b], c], b_{1}\right]=-\left[[[b, c], a], b_{1}\right]
$$

which we shall denote by $f$. Since $f$ is thus both in $B$ and in $A$ it commutes with both $B$ and $A$ and hence the ideal of $L$ that it generates is $\langle f\rangle+[f, L]$ $\leqslant\langle f\rangle+[f, C] \leqslant B$. Therefore this ideal is in $L_{1}$. Thus $\left[[[a, b] c], b_{1}\right]=$ $-\left[[[b, c], a], b_{1}\right]$ is in $L_{1}$. By the Jacobi identity applied to $[b, c], a$, and $b_{1}$, we get

$$
\left[[[b, c] a], b_{1}\right]+\left[\left[a, b_{1}\right],[b, c]\right]+\left[\left[b_{1},[b, c]\right], a\right]=0 .
$$

Since the third of these summands is 0 we get that $\left[\left[a, b_{1}\right],[b, c]\right]$ is in $L_{1}$. But this is the general form of the typical generator of $L^{\prime \prime}$; and hence $L^{\prime \prime} \leqslant L_{1}$ and is nilpotent of class at most 3 as was to be proved.

THEOREM 3. Let the group $G$ be generated by abelian subgroups $A, B, C$, such that $[A, B] \leqslant A,[B, C] \leqslant B,[C, A] \leqslant C$; then $G^{\prime \prime}$ is nilpotent of class at most 3.

Proof. Let $G_{1}=A_{1} B_{1} C_{1}$ where $A_{1}, B_{1}, C_{1}$ are the maximal normal subgroups of $G$ contained in $A, B, C$ respectively. Then (cf. [2, p. 177] for equations on commutator calculus)

$$
\begin{aligned}
G_{1}^{2} & =\left[G_{1}, G_{1}\right]=\left[A_{1} B_{1} C_{1}, A_{1} B_{1} C_{1}\right]=\left[A_{1}, B_{1}\right]\left[B_{1}, C_{1}\right]\left[C_{1}, A_{1}\right] \\
& \leqslant\left(A_{1} \cap B_{1}\right)\left(B_{1} \cap C_{1}\right)\left(C_{1} \cap A_{1}\right)
\end{aligned}
$$

and

$$
G_{1}^{3}=\left[G_{1}^{2}, G_{1}\right] \leqslant A_{1} \cap B_{1} \cap C_{1} \leqslant A \cap B \cap C
$$

which is central in $G$. Thus $G_{1}$ is nilpotent of class at most 3 .

Now for $a \in A, b \in B, c \in C$, we have from the Witt identity

$$
\left[\left[a, c^{-1}\right], b\right]^{c}\left[\left[c, b^{-1}\right], a\right]^{b}\left[\left[b, a^{-1}\right], c\right]^{a}=1
$$

with the first element $b_{0}=\left[\left[a, c^{-1}\right], b\right]^{c}$ of the product in $B$, the second element $a_{0} \in A$, and the third element $c_{0} \in C$. Thus $b_{0}=c_{0}^{-1} a_{0}^{-1}=c_{1} a_{1}$ with $c_{1}=c_{0}^{-1}, a_{1}=a_{0}^{-1}$.

For $b_{2} \in B$, we get $1=\left[b_{0}, b_{2}\right]^{a_{1}^{-1}}=\left[c_{1} a_{1}, b_{2}\right]^{a_{1}^{-1}}=\left[c_{1}, b_{2}\right]\left[a_{1}, b_{2}\right]^{a_{1}^{-1}}$. Then the element $\left[a_{1}, b_{2}\right]^{a_{1}^{-1}}=\left[b_{2}, c_{1}\right]$ is both in $A$ and in $B$ and its normal closure is in $B$. Thus $\left[a_{1}, b_{2}\right]^{b^{-1}}=\left[\left[a,\left[c, b^{-1}\right]\right], b_{2}\right]$ is in $G_{1}$.

If we now apply the Witt identity to the elements $a_{1}, b_{2},\left[c, b^{-1}\right]$ we get

$$
\left[\left[a, b_{2}^{-1}\right],\left[c, b^{-1}\right]\right]^{b_{2}}\left[\left[\left[c, b^{-1}\right], a^{-1}\right] b_{2}\right]^{a}=1
$$


(the second term of the expansion in the Witt identity is 1 since $b_{2}$ and $\left[c, b^{-1}\right]$ commute). Thus the elements of the form $\left[\left[a, b_{1}\right],\left[c, b_{2}\right]\right]$ are also in $G_{1}$.

We now observe that $G^{\prime}=[A B C, A B C]$ is generated by conjugates of $[a, b],[b, c]$ and $[c, a]$ with $a \in A, b \in B, c \in C$. Furthermore $\left[[a, b]^{8}\right.$, $\left.[b, c]^{h}\right]$ is in the normal subgroup $G_{1}$ if and only if $\left[[a, b]^{h^{-1}},[b, c]\right] \in G_{1}$. But $g h^{-1}=b_{0} a_{0} c_{0}^{-1}$ for suitable $a_{0} \in A, b_{0} \in B, C_{0} \in C$. Then $\left[[a, b]^{g h^{-1}},[b, c]\right]$ $\in G_{1}$ if and only if $\left[[a, b]^{b_{0} a_{0}},[b, c]\right]^{c_{0}} \in G_{1}$. But this latter element is $\left[\left[a_{1}, b^{b_{0} a_{0}}\right],\left[b_{1}, c\right]\right]$ with $a_{1}=a^{b_{0} a_{0}}$ and $b_{1}=b^{c_{0}}$; and this is $\left[\left[a_{1}, b\right],\left[b_{1}, c\right]\right]$ since $\left[a_{1}, b^{a_{0}}\right]=\left[a_{1},\left[a_{0}, b^{-1}\right], b\right]=\left[a_{1}, b\right]$ : We conclude that the generators of $G^{\prime \prime}$ are in $G_{1}$ and hence that $G^{\prime \prime}$ is nilpotent of class at most 3 as was to be shown.

\section{REFERENCES}

1. J. Mennicke, Einige endliche Gruppen mit drei Erzeugenden und drei Relationen, Arch. Math. (Basel) 10 (1959), 409-418.

2. Eugene Schenkman, Group theory, Krieger, Huntington, N.Y., 1975.

Department of Mathematics, Purdue University, West Lafayette, Indiana 47907 